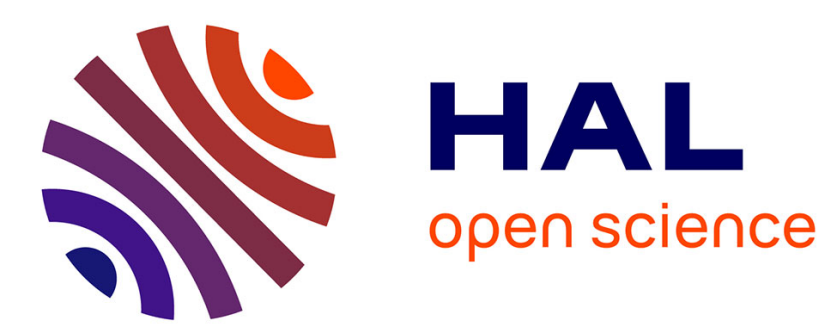

\title{
Sensitivity study of dynamic systems using polynomial chaos
}

\author{
Eduardo Haro Sandoval, Floriane Anstett-Collin, Michel Basset
}

\section{To cite this version:}

Eduardo Haro Sandoval, Floriane Anstett-Collin, Michel Basset. Sensitivity study of dynamic systems using polynomial chaos. Reliability Engineering and System Safety, 2012, 104, pp.15-26. 10.1016/j.ress.2012.04.001 . hal-00696271

\section{HAL Id: hal-00696271 https://hal.science/hal-00696271}

Submitted on 4 Jun 2012

HAL is a multi-disciplinary open access archive for the deposit and dissemination of scientific research documents, whether they are published or not. The documents may come from teaching and research institutions in France or abroad, or from public or private research centers.
L'archive ouverte pluridisciplinaire HAL, est destinée au dépôt et à la diffusion de documents scientifiques de niveau recherche, publiés ou non, émanant des établissements d'enseignement et de recherche français ou étrangers, des laboratoires publics ou privés. 


\title{
Sensitivity study of dynamic systems using polynomial chaos
}

\author{
Eduardo Haro Sandoval ${ }^{\mathrm{a}, *}$, Floriane Anstett-Collin ${ }^{\mathrm{b}, * *}$, Michel Basset $^{\mathrm{c}}$ \\ ${ }^{a}$ Universidad Panamericana, Augusto Rodin 498, 03920 México D.F. \\ ${ }^{b}$ Université de Lorraine, 2 rue Jean Lamour - 54500 Vandoeuvre Lès Nancy Cedex, France \\ ${ }^{c}$ Université de Haute Alsace, 12 rue des frères Lumière - 68093 Mulhouse Cedex, France
}

\begin{abstract}
Global sensitivity has mainly been analyzed in static models, though most physical systems can be described by differential equations. Very few approaches have been proposed for the sensitivity of dynamic models and the only ones are local. Nevertheless, it would be of great interest to consider the entire uncertainty range of parameters since they can vary within large intervals depending on their meaning. Other advantage of global analysis is that the sensitivity indices of a given parameter are evaluated while all the other parameters can be varied. In this way, the relative variability of each parameter is taken into account, revealing any possible interactions. This paper presents the global sensitivity analysis for dynamic models with an original approach based on the polynomial chaos (PC) expansion of the output. The evaluation of the PC expansion of the output is less expensive compared to direct simulations. Moreover, at each time instant, the coefficients of the PC decomposition convey the parameter sensitivity and then a sensitivity function can be obtained. The PC coefficients are determined using non-intrusive methods. The proposed approach is illustrated with some well-known dynamic systems.
\end{abstract}

Keywords: Global sensitivity analysis, dynamic system, polynomial chaos expansion, sensitivity functions

\section{Introduction}

Very often, the equations of a model involve unknown parameters which must be estimated from experimental data. A number of parameters are estimated with more or less precision, which may lead to unacceptable uncertainty on the model output. Among all the parameters, however, only few have a small

\footnotetext{
*Principal corresponding author. Tel.:(52) 5882 1600, Fax:(52) 54821700

** Corresponding author. Tel.: +33 (0)3 83685139, Fax: +33 (0)3 83685001

Corresponding author. Tel.: +33 (0)3 89336996, Fax: +33 (0)389423282

Email addresses: eharo@up.edu.mx (Eduardo Haro Sandoval),

floriane.collin@univ-lorraine.fr (Floriane Anstett-Collin)
} 
or insignificant influence on the model response and therefore do not need to be determined precisely. On the other hand, some parameters are decisive for the model response and thus influence its uncertainty significantly. These parameters may require additional measurement data in order to be estimated with relatively high accuracy. To prepare and plan the experiments, it is necessary to distinguish the parameters with an insignificant influence on the response uncertainty, so as to set them at their nominal value in their interval of variation, thanks to the sensitivity analysis. Numerous studies have focused on the sensitivity analysis for static nonlinear models, for example, [1, 2, 3, 4, 5, 6, 7]. The approaches may be local or global. Local approaches help to determine the impact of a small parameter variation around a nominal value [8]. Global approaches also allow the determination of the same impact but by varying the parameter in its entire range of variation. Global methods are often based on the analysis of the output variance and are known as ANOVA (ANalysis Of VAriance) techniques $[9,10,7]$. More recently, sensitivity moment-independent methods have been used, where emulation model is used to compute densitybased sensitivity measure [11]. The emulator is the one of [12].

The model function is split into a sum of functions of increasing dimension [7]. This decomposition, known as High Dimensional Model Representation (HDMR), performs the separation of the effects of different input parameters, which are transmitted in the decomposition of the variance. The present study exclusively focuses on global approaches. In order to quantify the contribution of a parameter to the output variance, a sensitivity index is calculated, often analytically when the model function is known and relatively simple. However, some models may be complex with a high number of parameters so that analytical calculations of the sensitivity indices become time consuming or even impossible. It is therefore necessary to estimate them $[3,13,14,15,7]$. Very often, they are computed using Monte Carlo simulations, but for computationally demanding models, this can become intractable. To overcome this drawback, the model of interest is replaced by an analytical approximation, called metamodel, for example, by polynomials which are less expensive. The sensitivity indices are then obtained straightforwardly from the algebraic expression of the coefficients of the polynomial expansion. The polynomial chaos (PC) decomposition is an example of such metamodelling. The PC expansion appeared in the 1930's as an effective means to represent stochastic processes in mechanics [16]. It is based on a probabilistic framework and represents amounts from stochastic spectral expansions of orthogonal polynomials $[17,18]$. It has recently been used in an original manner for sensitivity analysis purposes in [1, 19, 20, 21, 22, 23]. The great advantage of PC-based sensitivity approaches is that the full randomness of the response is contained in the set of the expansion coefficients.

On the other hand, the analysis of influential parameters is also important for dynamic models since most physical systems (biological, mechanical, electrical and so on) can be described by differential equations. Very few approaches have been proposed in the literature for the sensitivity analysis of dynamic models and the proposed ones are based on local derivatives or on one-at-a-time approaches [24, 25]. However, for some applications, mechanical or biological 
ones for instance, it can be of great importance to consider the entire uncertainty range of parameters since they can vary within large intervals depending on their meaning. Another advantage of global sensitivity analysis is that the sensitivity estimates of individual parameters are evaluated while all the other parameters are varied. In this way, the relative variability of each parameter is taken into account, thus revealing any existing interactions.

The global sensitivity analysis for dynamic models is addressed in this paper. In $[26,27]$, the PC expansion for stochastic differential equations has been studied to represent the model output and to get its statistic properties, but the parameter sensitivity has not been dealt with. Based on these studies, an original approach using the $\mathrm{PC}$ decomposition of the output is investigated here, to calculate the parameter sensitivity for dynamic models. At each time instant, the PC coefficients of the decomposition convey the parameter sensitivity and then a sensitivity function of each parameter can be obtained from the algebraic expression of the coefficients. The PC coefficients are determined either by regression or projection techniques which have the advantage of being nonintrusive methods. The proposed approach is illustrated with the well-known mass-spring-damper and DC motor systems.

The outline of this paper is as follows. The sensitivity functions for dynamic systems are presented in Section 2 and the PC expansion for the output of a differential equation in Section 3. Section 4 is focused on the determination of the PC coefficients. Section 5 proposes a PC-based approach to the estimation of the sensitivity functions, which approach is summed up in Section 6. Finally, Section 7 presents an analytical test case to show the convergence of the numerical results. Moreover, the provided approach is applied on some representative dynamic physical systems.

\section{Global sensitivity analysis}

Consider the following stochastic differential equation:

$$
\mathcal{L}(t, \omega, \mathbf{p}(\omega) ; y)=f(t, \omega, \mathbf{p}(\omega))
$$

where $\mathcal{L}$ is a linear or non-linear differential operator and $\mathbf{p}(\omega)=\left(p_{1}(\omega), \ldots, p_{n}(\omega)\right)$ with $p_{i}(\omega), i=1, \ldots, n$, the $n$ unknown parameters, considered as uniformly random and independent variables, defined on the unit cube $K$. The stochastic variable $\omega$ is used to indicate the randomness of the input variable p. For the sake of simplicity, $\omega$ will be omitted in the following and $\mathbf{p}$ stands for $\mathbf{p}(\omega)$. The solution $y=y(t, \mathbf{p})$, corresponding to the output of the model, can be decomposed into summands of increasing dimension [7], at each time instant:

$$
\begin{aligned}
y(t, \mathbf{p})= & f_{0}(t)+\sum_{i=1}^{n} f_{i}\left(t, p_{i}\right)+\sum_{i=1}^{n-1} \sum_{i<j}^{n} f_{i j}\left(t, p_{i}, p_{j}\right) \\
& +\ldots+f_{1 \ldots n}\left(t, p_{1}, \ldots, p_{n}\right)
\end{aligned}
$$

where $y(t, \mathbf{p}) \in \mathbb{R}$ the model output is assumed continuous, derivative and square-integrable. The term $f_{0}(t)$ is the mean value of the output at each 
time instant:

$$
f_{0}(t)=\int_{K^{n}} y(t, \mathbf{p}) \mathrm{d} \mathbf{p}
$$

The summands of equation (2) are given by:

$$
\begin{aligned}
& f_{i}\left(t, p_{i}\right)=E\left[y(t, \mathbf{p}) \mid p_{i}\right]-f_{0}(t) \\
& f_{i j}\left(t, p_{i}, p_{j}\right)=E\left[y(t, \mathbf{p}) \mid p_{i}, p_{j}\right]-f_{i}(t)-f_{j}(t)-f_{0}(t) \\
& \ldots
\end{aligned}
$$

where $E\left[y(t, \mathbf{p}) \mid p_{i}\right]$ (resp. $E\left[y(t, \mathbf{p}) \mid p_{i}, p_{j}\right]$ ) is the conditional expectation of $y(t, \mathbf{p})$ when $p_{i}$ is set (resp. $p_{i}$ and $p_{j}$ are set).

The integral of each summand $f_{i_{1} \ldots i_{s}}\left(t, p_{i_{1}}, \ldots, p_{i_{s}}\right)$ is zero:

$$
\int_{0}^{1} f_{i_{1} \ldots i_{s}}\left(t, p_{i_{1}}, \ldots, p_{i_{s}}\right) \mathrm{d} p_{i_{k}}=0
$$

with $k \in\left\{i_{1}, \ldots, i_{s}\right\}$ and $1 \leq i_{1} \leq \ldots \leq i_{s} \leq n$. Due to equation (5), the summands are orthogonal to each other:

$$
\int_{K^{n}} f_{i_{1} \ldots i_{s}}\left(t, p_{i_{1}}, \ldots, p_{i_{s}}\right) f_{j_{1} \ldots j_{r}}\left(t, p_{j_{1}}, \ldots, p_{j_{r}}\right) \mathrm{d} \mathbf{p}=0
$$

for $\left\{i_{1}, \ldots, i_{s}\right\} \neq\left\{j_{1} \ldots, j_{r}\right\}$.

There are infinite possible decompositions but only one is satisfying Sobol' orthonormality condition (5).

Moreover, the variance of the output, denoted $V(t)$, is given by:

$$
V(t)=\int_{K^{n}}\left(y^{2}(t, \mathbf{p})-f_{0}^{2}(t)\right) \mathrm{d} \mathbf{p}
$$

The decomposition (2) leads to the following decomposition of the variance $V(t)$ :

$$
V(t)=\sum_{i=1}^{n} V_{i}(t)+\sum_{i=1}^{n-1} \sum_{i<j}^{n} V_{i j}(t)+\ldots+V_{1 \ldots n}(t)
$$

with:

$$
\begin{aligned}
& V_{i}(t)=V\left[E\left[y(t, \mathbf{p}) \mid p_{i}\right]\right] \\
& V_{i j}(t)=V\left[E\left[y(t, \mathbf{p}) \mid p_{i}, p_{j}\right]\right]-V_{i}(t)-V_{j}(t) \\
& \vdots \\
& V_{1 \ldots n}(t)=V(t)-\sum_{i=1}^{n} V_{i}(t)-\sum_{1 \leq i<j \leq n} V_{i j}(t)-\cdots-\sum_{1 \leq i_{1}<\ldots<i_{n-1} \leq n} V_{i_{1} \ldots i_{n-1}}(t)
\end{aligned}
$$

where $V\left[E\left[y(t, \mathbf{p}) \mid p_{i}\right]\right]$ (resp. $V\left[E\left[y(t, \mathbf{p}) \mid p_{i}, p_{j}\right]\right]$ ) is the variance of the conditional expectation of $y(t, \mathbf{p})$ when $p_{i}$ is set (resp. $p_{i}$ and $p_{j}$ are set).

Since the output $y(t, \mathbf{p})$ varies with time, there is one value for the classic Sobol 
sensitivity indices at each time instant, thus leading to sensitivity functions. In the same manner as for static models, the sensitivity functions of parameter $p_{i}$ are obtained by renormalising (9) with the total variance $V(t)$. Thus, the first order sensitivity function, denoted $S_{i}(t)$, is defined as follows:

$$
S_{i}(t)=\frac{V_{i}(t)}{V(t)}
$$

The first order sensitivity function $S_{i}(t)$ represents the main effect of the parameter $p_{i}$ which corresponds to its contribution alone. The value of $S_{i}(t)$, at each time instant, lies between 0 and 1 . The closer to 1 its value is, the more parameter $p_{i}$ contributes to the total variance of the output. The sensitivity functions of higher orders, denoted $S_{i_{1} \ldots i_{s}}(t)$, are defined as:

$$
S_{i_{1} \ldots i_{s}}(t)=\frac{V_{i_{1} \ldots i_{s}}(t)}{V(t)}, \quad 1 \leq i_{1} \leq \ldots \leq i_{s} \leq n
$$

The sensitivity functions $S_{i_{1} \ldots i_{s}}(t)$ represent the collective contribution of the parameters $p_{i_{1}}, \ldots, p_{i_{s}}$, which corresponds to the influence of the interactions of these parameters. By normalising equation (8) with the total variance $V(t)$, the following expression is obtained:

$$
1=\sum_{i=1}^{n} S_{i}(t)+\sum_{i=1}^{n-1} \sum_{i<j}^{n} S_{i j}(t)+\ldots+S_{1 \ldots n}(t)
$$

The total sensitivity function of parameter $p_{i}$, denoted $S_{T i}(t)$, represents the contribution of $p_{i}$ alone in addition to the contribution due to its interaction with the other parameters [9]. This total sensitivity function is defined by:

$$
S_{T i}(t)=S_{i}(t)+\sum_{\substack{j=1 \\ j \neq i}}^{n} S_{i j}(t)+\sum_{\substack{j=1 \\ j \neq i}}^{n} \sum_{\substack{k=j+1 \\ k \neq i \\ j \neq i}}^{n} S_{i j k}(t)+\ldots+S_{i j k \ldots n}(t)
$$

The previous sensitivity functions can be calculated using Monte Carlo simulations. However, the number of model evaluations can become very large and the calculations intractable, especially as they have to be performed at each time instant. To overcome this problem, the model response is substituted by its PC expansion, which is an analytical representation in an orthonormal polynomial basis. Therefore, evaluating the PC expansion of the output is less expensive compared to direct simulations. Furthermore, the sensitivity functions can be evaluated as analytical expressions of the PC coefficients. In the next section, the PC expansion of the model output is detailed.

\section{PC expansion of the model output}

The beginnings of the polynomial chaos can be traced back to Wiener [16]. He suggested that the spectral expansion of Hermite polynomials in terms of 
Gaussian random variables could be used to represent certain stochastic processes. It was then shown that the homogeneous chaos expansion could be used to approximate any function in the Hilbert space of square-integrable functions. As a result, any second-order random variable with finite variance can be represented by a spectral expansion standing on a basis of Hermite polynomials. In $[26,27]$, this is extended to differential equations. In this case, any second-order random variable $X(t, \omega)$ can be expanded as:

$$
X(t, \omega)=\sum_{j=0}^{\infty} x_{j}(t) \Phi_{j}(\xi(\omega))
$$

where $x_{j}(t)$ are unknown deterministic coefficients, $\xi(\omega)=\left(\xi_{1}(\omega), \ldots, \xi_{n}(\omega)\right)$ the $n$ standard normal independent random variables (zero mean and unit standard deviation) and $\Phi_{j}$ the multivariate Hermite polynomials. The basis is orthogonal with respect to the Gaussian measurement. As previously, for the sake of simplicity, $\omega$ will be omitted and $\xi$ stands for $\xi(\omega)$.

In practice, the PC expansion (14) is truncated to a finite number $M+1$ of terms, i.e. the coefficients to be calculated. The degree $d$ of the polynomials is related to $M$ and to the number $n$ of random variables $\xi$ by:

$$
M+1=\frac{(n+d) !}{n ! d !}
$$

It is worth noting that the required degree for the polynomials is not known a priori and must be tested to obtain the best accuracy. The multivariate polynomial $\Phi_{j}$ is given by the tensor product of the corresponding one-dimensional Hermite polynomials $\phi_{a_{k}^{j}}$ :

$$
\Phi_{j}(\xi)=\prod_{k=1}^{n} \phi_{a_{k}^{j}}\left(\xi_{k}\right)
$$

with $a_{k}^{j}$ the degree of the one-dimensional Hermite polynomials, such that $\left|a^{j}\right|=\sum_{k=1}^{n} a_{k}^{j} \leq d, j=0, \ldots, M$.

The Hermite expansion above has been used effectively for equations with Gaussian inputs. But, according to the theorem of Cameron and Martin [28], this function converges for arbitrary random processes. For example, normalised Legendre polynomials can be associated with a uniform distribution. Table 1 sums up the type of distribution and the associated polynomials.

Assuming that the model output $y(t, \mathbf{p})$ is square-integrable and the parameters independent, $y(t, \mathbf{p})$ can also be decomposed in $\mathrm{PC}$ :

$$
y(t, \mathbf{p}) \approx \sum_{j=0}^{M} \alpha_{j}(t) \Phi_{j}(\mathbf{p}), \quad \Phi_{j}(\mathbf{p})=\prod_{k=1}^{n} \phi_{a_{k}^{j}}\left(p_{k}\right)
$$




\begin{tabular}{|c|c|c|c|}
\hline & Distribution & Polynomials & Support \\
\hline \hline Continuous & Gaussian & Hermite & $\mathbb{R}$ \\
\hline & Uniform & Legendre & {$[-1 ; 1]$} \\
\hline & Gamma & Laguerre & {$[0 ;+\infty[$} \\
\hline & Beta & Jacobi & {$[-1 ; 1]$} \\
\hline Discrete & Poission & Charlier & $\{0,1, \ldots\}$, \\
\hline & Negative binomial & Miexner & $\{0,1, \ldots\}$, \\
\hline & Binomial & Krawtchouk & $\{0,1, \ldots, N\}$ \\
\hline & Hypogeometric & Hahn & $\{0,1, \ldots, N\}$ \\
\hline
\end{tabular}

Table 1: Wiener-Askey polynomials

As stated in Section 2, the model parameters $\mathbf{p}$ are uniformly independent random variables. Therefore, the one-dimensional polynomials $\phi_{a_{k}^{j}}$ in (17) are Legendre polynomials, defined by the following recurrence equation:

$$
\left\{\begin{array}{l}
\phi_{0}\left(p_{k}\right)=1 \\
\phi_{i+1}\left(p_{k}\right)=\left(\frac{2 i+1}{i+1}\right) p_{k} \phi_{i}\left(p_{k}\right)-\left(\frac{i}{i+1}\right) \phi_{i-1}\left(p_{k}\right), \quad \text { for } i>1
\end{array}\right.
$$

Moreover, Table 1 emphasizes that the Legendre polynomials are orthogonal with respect to a uniform probability measurement over $[-1 ; 1]$, while the parameters $p_{k}, k=1, \ldots, n$, lies in $[0 ; 1]$. Consequently, the parameters are first mapped onto the interval $[-1 ; 1]$, before completing the PC expansion.

The deterministic coefficients $\alpha_{j}(t), j=1, \ldots, M$, are unknown. The next section is focused on their determination.

\section{Determination of the PC coefficients}

Once the structure of the PC expansion is obtained for the model output, as explained in the previous section, the deterministic coefficients $\alpha_{j}(t)$ must be computed. There exist two types of methods for this, the intrusive and the non-intrusive ones. Historically, the intrusive Stochastic Galerkin (SG) method was used $[29,27,28]$. A Galerkin projection helps to minimize the error of the truncated expansion, and all the resulting coupled equations are solved for the coefficients of the expansion equations. SG method is well suited for the treatment of ordinary and partial differential equations as it has the ability to cope with a large nonlinear dependence of the solution on data from random patterns. However, SG method is intrusive in the sense that it must solve a system of coupled equations which require robust and efficient algorithms and the modification of an existing deterministic code. Often, the form of equations or code used to solve the deterministic equations is complex and it makes the implementation of the PC difficult, if not impossible. Therefore, non-intrusive methods have been proposed based on regression technique $[30,31]$ or on projection method $[19,20]$. Instead of building prior expansions of uncertainty sources 
and of finding the coefficients by solving the corresponding equations, these approaches use interpolation methods and project a set of deterministic simulations, which are defined using samples of parameters chosen carefully, with a polynomial basis. These approaches are very useful when trying to quantify uncertainty in complex deterministic models which cannot easily be modified. Similarly to SG methods, non-intrusive methods reach a fast convergence when the solutions are sufficiently smooth in the random space.

\subsection{Regression method}

The PC coefficients are obtained through the regression of the mean-square optimization problem. In the following, consider a sample of size $N$ for the output $y$ and the parameters $\mathbf{p}$, at each time instant. The $k$-th realisation of $\mathbf{p}$ and of $y$, at time $t$, is denoted, respectively, by $\mathbf{p}^{(k)}$ and $y^{(k)}\left(t, \mathbf{p}^{(k)}\right)$. The mean-square minimization helps to solve the following problem:

$$
\hat{\alpha}(t)=\arg \min \frac{1}{N} \sum_{k=1}^{N}\left[y^{(k)}\left(t, \mathbf{p}^{(k)}\right)-\sum_{j=0}^{M} \alpha_{j}(t) \Phi_{j}\left(\mathbf{p}^{(k)}\right)\right]^{2}
$$

where $\hat{\alpha}(t)=\left(\hat{\alpha}_{0}(t), \ldots, \hat{\alpha}_{M}(t)\right)$ is the vector of PC coefficients. The notation $\hat{\alpha}(t)$ is employed to distinguish the estimated value of $\alpha(t)$ from its true one. Denoting by $\boldsymbol{\Phi}$ the matrix whose elements are given by $\boldsymbol{\Phi}_{k j}=$ $\Phi_{j}\left(\mathbf{p}^{(k)}\right), k=1, \ldots, N$ and $j=0, \ldots, M$, by $\boldsymbol{\Phi}^{T}$ its transpose and by $\mathbf{y}(t)=$ $\left(y^{(1)}\left(t, \mathbf{p}^{(1)}\right), \ldots, y^{(N)}\left(t, \mathbf{p}^{(N)}\right)\right.$ the vector containing the $N$ realisations of $y$, at time $t$, the solution of (19) is:

$$
\hat{\alpha}(t)=\left(\boldsymbol{\Phi}^{T} \boldsymbol{\Phi}\right)^{-1} \boldsymbol{\Phi}^{T} \mathbf{y}(t)
$$

It is generally advisable to use an over-sampling to determine $N$, resulting in a least squares solution for the over-determined system. It can be calculated by $N=2(M+1)$ [32], or $N=(n-1)(M+1)$ [20]. As stated in [20,33], taking more points does not improve the accuracy of the results.

\subsection{Projection method}

The projection method takes advantage of the orthogonality properties of the $\mathrm{PC}$ representation computing the expansion coefficients from some evaluations of the model. Multiplying both sides of equation (17) by $\Phi_{l}(\mathbf{p})$ and taking the inner product, it reads:

$$
\left\langle y(t, \mathbf{p}), \Phi_{l}(\mathbf{p})\right\rangle \approx \sum_{j=0}^{M} \alpha_{j}(t)\left\langle\Phi_{j}(\mathbf{p}), \Phi_{l}(\mathbf{p})\right\rangle
$$

where $\langle\cdot, \cdot\rangle$ is the inner product in the Hilbert space. Orthogonality property is now used. Indeed, polynomials $\Phi_{j}(\mathbf{p})$ form an orthogonal basis so that:

$$
\left\langle\Phi_{j}(\mathbf{p}), \Phi_{l}(\mathbf{p})\right\rangle=\delta_{j l}
$$


where $\delta_{j l}$ is the Delta Kronecker symbol, $\delta_{j l}=1$ if $j=l$ and 0 otherwise. Thus, from (21), the coefficients $\hat{\alpha}_{j}(t)$ can be obtained:

$$
\hat{\alpha}_{j}(t)=\frac{\left\langle y(t, \mathbf{p}), \Phi_{j}(\mathbf{p})\right\rangle}{\left\langle\Phi_{j}(\mathbf{p}), \Phi_{j}(\mathbf{p})\right\rangle}, \quad \forall j
$$

In the latter expression the denominator is already known and the numerator can be computed with the multidimensional integral:

$$
\left\langle y(t, \mathbf{p}), \Phi_{j}(\mathbf{p})\right\rangle=\int_{K^{n}} y(t, \mathbf{p}) \Phi_{j}(\mathbf{p}) h(\mathbf{p}) d \mathbf{p}
$$

where $h(\mathbf{p})$ is the joint probability density function of $\mathbf{p}$. In order to obtain the $j$-th coefficients, it can be shown that $M+1$ multidimensional integrals must be solved. There exist different methods to numerically compute (24), random sampling, quadrature product and Smolyak sparse grids.

\subsubsection{Random sampling}

The Random sampling is the Monte Carlo approach. Here the integral evaluation is equivalent to compute the mean of the function product (left member of equation (24)) for each term in the expansion. To have appropriate estimates a total-order chaos expansion must be used and the samples must have the density of the weighting function. In practice, this method can be intractable because the number of samples required for acceptable convergence can be large.

\subsubsection{Quadrature}

Consider the use of a numerical integration technique to estimate the coefficients of the expansion. The integral of (24) can be computed as a finite weighted summation evaluated at special points (the integration points). The theory of quadrature approaches is based on the use of orthogonal polynomials

of the $\mathrm{PC}$ basis. This numerical integration method can be expressed in the form:

$$
\int_{D^{n}} y(t, \mathbf{p}) \Phi_{j}(\mathbf{p}) h(\mathbf{p}) d \mathbf{p} \approx \sum_{k=1}^{N} y\left(t, \mathbf{p}^{(k)}\right) \Phi_{j}\left(\mathbf{p}^{(k)}\right) W^{(k)}
$$

with $\mathbf{p}^{(k)}$ being the integration points, $W^{(k)}$ the weights and $N$ the number of integration points. These methods require the smoothness of the function.

The Gauss-Hermite integration of this equation requires $N^{n}$ computations, where $n$ is the number of random variables. As for the number of the integration points, a minimal Gaussian quadrature order of $N=d+1$ will be required to obtain a good accuracy in the coefficients, where $d$ is the degree of the polynomials $[19,32,29,20]$. 


\subsubsection{Smolyak sparse grids}

When the number of parameters is very large and the number of evaluations of the integral (25) becomes impractical, a solution is to use Smolyak cubature $[19,32]$. Smolyak formulas are linear combinations of the products expressed in equation (25), which only takes into account products with relatively few integration points. These methods are based on the differences between quadratures of successive levels. The one-dimensional quadrature formula of level $l$, with points $p$ and weights $W$, respectively, writes:

$$
\int_{D^{n}} y(t, \mathbf{p}) \Phi_{j}(\mathbf{p}) h(\mathbf{p}) d \mathbf{p} \approx \sum_{k=1}^{N} y\left(t, \mathbf{p}^{(k)}\right) \Phi_{j}\left(\mathbf{p}^{(k, l)}\right) W^{(k, l)} \approx \mathcal{U}_{l}^{1}
$$

Setting $\mathcal{U}_{0}^{1}=0$ and with $i \geq 1$ an increasing sequence of integration formulas, the difference quadrature formula is defined as:

$$
\Delta_{i \geq 1}^{1}=\mathcal{U}_{i}^{1}-\mathcal{U}_{i-1}^{1}
$$

With a set of multi-indices $\mathbf{i}=\left(i_{1}, \ldots, i_{n}\right)$, the $n$-dimensional difference formula is the tensor product of one-dimensional difference quadrature. Then, the $n$ dimensional Smolyak's cubature formula of level $l$ is constructed by the sum of tensor products of difference quadratures, over a set of multi-indices i:

$$
\int_{D^{n}} y(t, \mathbf{p}) \Phi_{j}(\mathbf{p}) h(\mathbf{p}) d \mathbf{p} \approx \sum_{|\mathbf{i}| \leq l+n-1}\left(\Delta^{i_{1}} \otimes \cdots \otimes \Delta^{i_{n}}\right)
$$

\section{PC-based sensitivity functions}

Consider the PC expansion of the model output (17) with the coefficients computed with (19) or (23). Due to the orthogonality of the basis, it can be shown that the mean $\hat{\bar{y}}(t)$ and the variance $\hat{V}(t)$ of the output are, respectively, given by:

$$
\begin{gathered}
\hat{\bar{y}}(t)=\hat{\alpha}_{0}(t) \\
\hat{V}(t)=\sum_{j=1}^{M} \hat{\alpha}_{j}^{2}(t)\left\langle\Phi_{j}(\mathbf{p}), \Phi_{j}(\mathbf{p})\right\rangle
\end{gathered}
$$

As explained in [34], to compute the sensitivity function $S_{i}(t)(10)$ of parameter $p_{i}$, it is necessary to reorder the expansion (17) in order to separate the different contributions - single and collective - of each parameter, as (2). To do so, $\mathcal{I}_{k_{1}, \ldots, k_{s}}$ must define the set of multi-indices $j$ such that :

$$
\mathcal{I}_{k_{1}, \ldots, k_{s}}=\left\{0 \leq a_{k}^{j} \leq d, a_{k}^{j}=0 \forall k \in\{1, \ldots, n\} \backslash\left\{k_{1}, \ldots, k_{s}\right\}\right\}
$$


where $a_{k}^{j}$ is the one-dimensional polynomial degree. The set $\mathcal{I}_{i}$ corresponds to the polynomials depending on parameter $p_{i}$ only, with the exception of all the others. Using this notation, the expansion (17) can be written as:

$$
\begin{aligned}
& y(t, \mathbf{p}) \approx \alpha_{0}(t) \Phi_{0}(\mathbf{p})+\sum_{i=1}^{n} \sum_{j \in \mathcal{I}_{i}} \alpha_{j}(t) \Phi_{j}\left(p_{i}\right) \\
& +\sum_{\substack{1 \leq i_{1} \leq i_{2} \leq n \\
\sum_{1 \leq i_{1} \leq \ldots \leq i_{2} \leq n}}} \sum_{j \in \mathcal{I}_{i_{1}, i_{2}}} \alpha_{j}(t) \Phi_{j}\left(p_{i_{1}}, p_{i_{2}}\right)+\cdots \\
& +\cdots
\end{aligned}
$$

The first-order sensitivity function can be obtained straightforwardly from (32) with the estimated coefficients $\hat{\alpha}_{i}(t)$ (equation (19) or (23)). Thus, the estimated first-order sensitivity function $\hat{S}_{i}(t)$ of parameter $p_{i}$ is given by:

$$
\hat{S}_{i}(t)=\frac{\sum_{j \in \mathcal{I}_{i}} \hat{\alpha}_{j}^{2}(t)\left\langle\Phi_{j}\left(p_{i}\right), \Phi_{j}\left(p_{i}\right)\right\rangle}{\sum_{j=1}^{M} \hat{\alpha}_{j}^{2}(t)\left\langle\Phi_{j}(\mathbf{p}), \Phi_{j}(\mathbf{p})\right\rangle}
$$

The estimated sensitivity functions of a higher order, $\hat{S}_{i_{1}, \ldots, i_{s}}(t)$, reflecting the interactions effect, can be obtained in the same manner:

$$
\hat{S}_{i_{1}, \ldots, i_{s}}(t)=\frac{\sum_{j \in \mathcal{I}_{i_{1}, \ldots, i_{s}}} \hat{\alpha}_{j}^{2}(t)\left\langle\Phi_{j}\left(p_{i_{1}}, \ldots, p_{i_{s}}\right), \Phi_{j}\left(p_{i_{1}}, \ldots, p_{i_{s}}\right)\right\rangle}{\sum_{j=1}^{M} \hat{\alpha}_{j}^{2}(t)\left\langle\Phi_{j}(\mathbf{p}), \Phi_{j}(\mathbf{p})\right\rangle}
$$

Finally, the estimated total sensitivity function, $\hat{S}_{T i}(t)$ is given by:

$$
\hat{S}_{T i}(t)=\frac{\sum_{j \in \mathcal{I}_{i+}} \hat{\alpha}_{j}^{2}(t)\left\langle\Phi_{j}\left(p_{i}\right), \Phi_{j}\left(p_{i}\right)\right\rangle}{\sum_{j=1}^{M} \hat{\alpha}_{j}^{2}(t)\left\langle\Phi_{j}(\mathbf{p}), \Phi_{j}(\mathbf{p})\right\rangle}
$$

with $\mathcal{I}_{i^{+}}$the set of multi-indices $j$ defined by:

$$
\mathcal{I}_{i^{+}}=\left\{0 \leq a^{j} \leq p, \quad a_{i}^{j} \neq 0, \quad \forall k=i\right\}
$$

The set $\mathcal{I}_{i+}$ corresponds to polynomials $\Phi_{i}$ depending on parameter $p_{i}$ and possibly on the other parameters.

The great advantage of using the PC-based approach to the computation of 
the sensitivity functions is that the calculation is straightforward once the PC expansion is obtained. Then, this approach will be efficient if the PC expansion is accurate and inexpensive.

The next section gives a summary of the proposed approach.

\section{Summary of the proposed approach}

The computation of the sensivity functions of the parameters for the dynamic model (2), requires the following steps:

\section{a) PC decomposition}

\section{Polynomial type}

Choose the polynomial type associated to the parameter distribution, see Table 1.

If necessary, map the parameters onto the required interval given in Table 1 , in order to get an orthogonal basis for the chosen polynomials.

2. PC degree

Set the required degree $d$ for the PC.

3. PC truncation

Compute the number $M+1$ of terms in the expansion (17), according to (15).

4. $\mathrm{PC}$ coefficients

Compute the PC coefficients according to (19) or (23).

\section{b) Sensitivity functions}

Once the PC decomposition of the output is completed, compute the sensitivity functions according to (33), (34) and (35), for each parameter.

\section{Application examples}

A simple test case is provided in order to compute analytically the sensitivity indices and to show the convergence of the numerical results obtained using the PC decomposition. Then, two examples of dynamic system are given to illustrate the contribution of this work.

\subsection{Analytical test case}

Consider the solution $y(t)$ of a simple first-order differential equation of the form $\dot{y}(t)=p_{1}$ :

$$
y(t)=p_{1} t+p_{2}
$$

The parameters $p_{1}$ and $p_{2}$ are assumed to be independent uniformly distributed over $\left[c_{1} ; d_{1}\right]$ and $\left[c_{2} ; d_{2}\right]$, respectively. 


\subsubsection{Analytical sensitivity indices}

The mean value $\bar{y}(t)$ of the output is given by (3) and can be calculated analytically:

$$
\bar{y}(t)=\frac{c_{2}+d_{2}}{2}+\frac{\left(c_{1}+d_{1}\right) t}{2}
$$

The output variance $V(t)$ is given by (7) and its analytical expression is:

$$
V(t)=\frac{\left(c_{2}-d_{2}\right)^{2}}{12}+\frac{\left(c_{1}-d_{1}\right)^{2} t^{2}}{12}
$$

The first-order sensitivity functions $S_{p_{1}}(t)$ and $S_{p_{2}}(t)$ of the parameters $p_{1}$ and $p_{2}$ are given by (10) and their analytical expressions are, respectively:

$$
\begin{aligned}
& S_{p_{1}}(t)=\frac{\left(c_{1}-d_{1}\right)^{2} t^{2}}{\left(c_{2}-d_{2}\right)^{2}+\left(c_{1}-d_{1}\right)^{2} t^{2}} \\
& S_{p_{2}}(t)=\frac{\left(c_{2}-d_{2}\right)^{2}}{\left(c_{2}-d_{2}\right)^{2}+\left(c_{1}-d_{1}\right)^{2} t^{2}}
\end{aligned}
$$

It can be worth noting that model (37) is additive. Thus, the second-order index $S_{p_{1} p_{2}}(t)$ is zero and the total indices, $S_{T p_{1}}(t)$ and $S_{T p_{2}}(t)$, are equal to the first-order indices, $S_{p_{1}}(t)$ and $S_{p_{2}}(t)$, respectively.

\section{2. $P C$-based sensitivity indices}

Here, the number of parameters is $n=2$ and the degree used for the polynomials is $d=1$ as the output is linear in the parameters. Thus, the number of coefficients to compute in the decomposition is $M+1=3(15)$. The multivariate polynomials $\Phi_{j}$ are given by (16):

$$
\Phi_{j}\left(p_{1}, p_{2}\right)=\prod_{k=1}^{2} \phi_{a_{k}^{j}}\left(p_{k}\right)
$$

where $\left|a^{j}\right|=\sum_{k=1}^{2} a_{k}^{j} \leq 1, j=0, \ldots, 2$, and $\phi_{a_{k}^{j}}$ are one-dimensional Legendre polynomials. The mean value $\hat{\bar{y}}(t)$, the variance $\hat{V}(t)$ and the sensitivity functions $\hat{S}_{p_{1}}(t)$ and $\hat{S}_{p_{2}}(t)$ are given, respectively, by (29), (30), (33), (34) and (35). In the following, the numerical values are $c_{1}=0.76, d_{1}=0.84, c_{2}=0.9$ and $d_{2}=1.1$. The figure 1 represents the evolution of the output variance computed analytically vs using PC decomposition (top figure). The relative error, shown in figure 1 (down), is given, in percentage, by $\frac{100|V(t)-\hat{V}(t)|}{V(t)}$. This error does not exceed $6 \%$, showing good accuracy of the results.

The figures 2 and 3 represent, respectively, the sensitivity functions of $p_{1}$ and $p_{2}$, computed analytically vs using PC decomposition (top) and the relative error of the sensitivity functions (down). These figures show that the relative errors, computed as previously, do not exceed $3 \%$ for $p_{1}$ and $7.5 \%$ for $p_{2}$, showing satisfactory accuracy of the PC decomposition. 

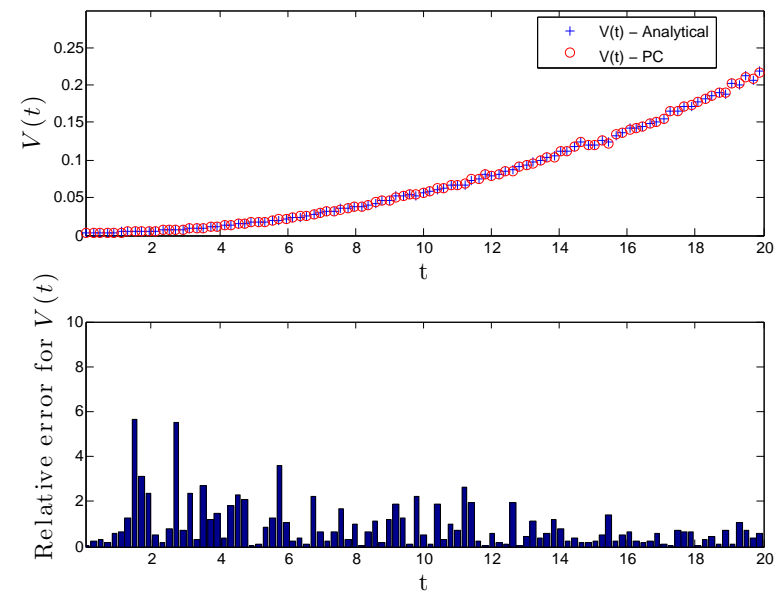

Figure 1: Evolution of the output variance (top) - Relative error for the output variance $V(t)$ (down)

\subsection{Mass-spring-damper system}

Also to illustrate the contribution of this paper, an ideal mass-spring-damper system is considered, with a mass $m$, a spring constant $b$ and a viscous damping coefficient $k$, subject to a constant force $F$, as represented in figure 4 .

This system is described by the following differential equation:

$$
m \ddot{y}(t)+b \dot{y}(t)+k y(t)=F
$$

where $y(t)$, the output of the system, represents the mass position. This equation can be represented by a state space system. Defining the states as 

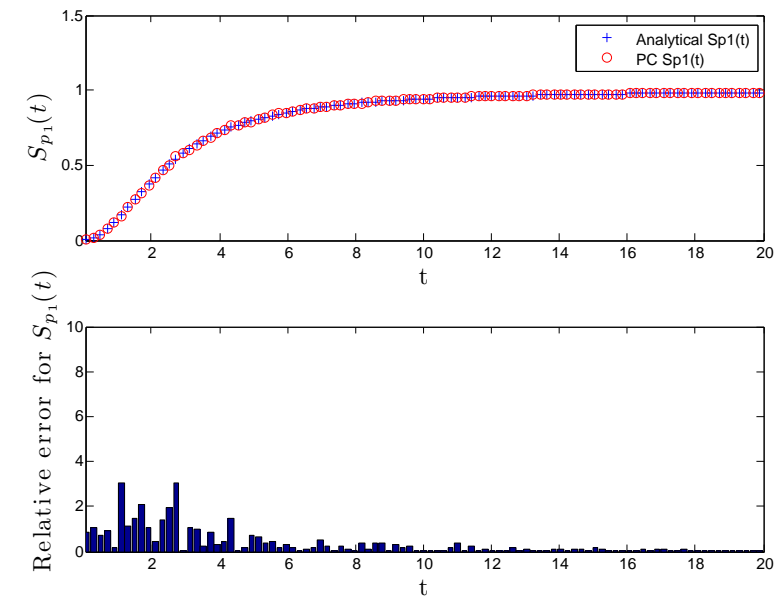

Figure 2: Sensitivity function $S_{p_{1}}(t)$ (top) - Relative error for $S_{p_{1}}(t)$ (down)

$x_{1}(t)=y(t)$ and $x_{2}(t)=\dot{y}(t)$, it reads:

$$
\left[\begin{array}{l}
\dot{x}_{1}(t) \\
\dot{x}_{2}(t)
\end{array}\right]=\left[\begin{array}{cc}
0 & 1 \\
-\frac{k}{m} & -\frac{b}{m}
\end{array}\right]\left[\begin{array}{l}
x_{1}(t) \\
x_{2}(t)
\end{array}\right]+\left[\begin{array}{c}
0 \\
\frac{1}{m}
\end{array}\right] F
$$

Figure 5 is the simulation when a step signal is applied on $F$, for the fixed values $m=1, b=1$ and $k=4.5$.

The sensitivity of the parameters $m, b$ and $k$ on the mass position $y(t)$ is considered. The proposed approach is applied in order to obtain the sensitivity functions. 

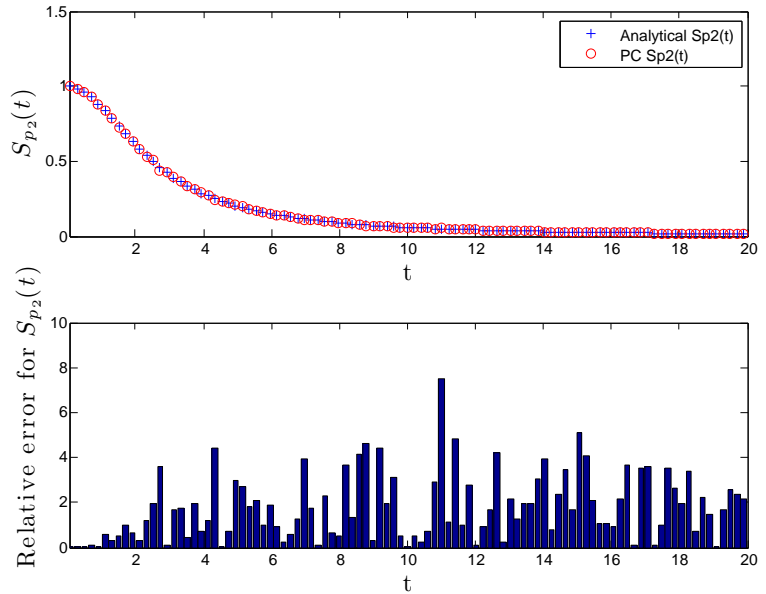

Figure 3: Sensitivity function $S_{p_{2}}(t)$ (top) - Relative error for $S_{p_{2}}(t)$ (down)

\subsubsection{PC decomposition}

1. Polynomial type

The parameters $p_{1}=m, p_{2}=b$ and $p_{3}=k$ are considered as independent random variables with a uniform distribution in the following intervals, $p_{1} \in[0.9 ; 1.1], p_{2} \in[0.8 ; 1.2]$ and $p_{3} \in[3 ; 6]$. The first step is to decompose the output $y(t)$ in $\mathrm{PC}$ (equation (17)). As the parameters are uniformly distributed, $\phi_{a_{k}^{j}}$ are Legendre polynomials.

Before starting the decomposition, the parameters must be mapped onto 


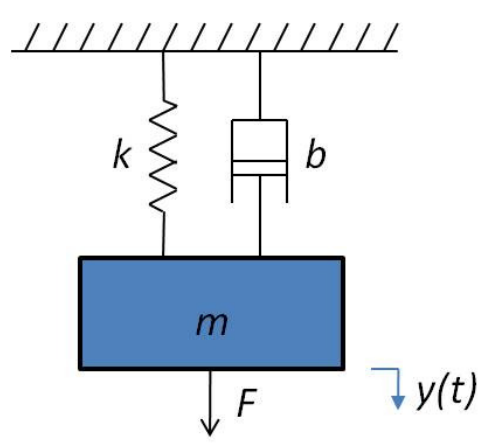

Figure 4: Mass-spring damper system

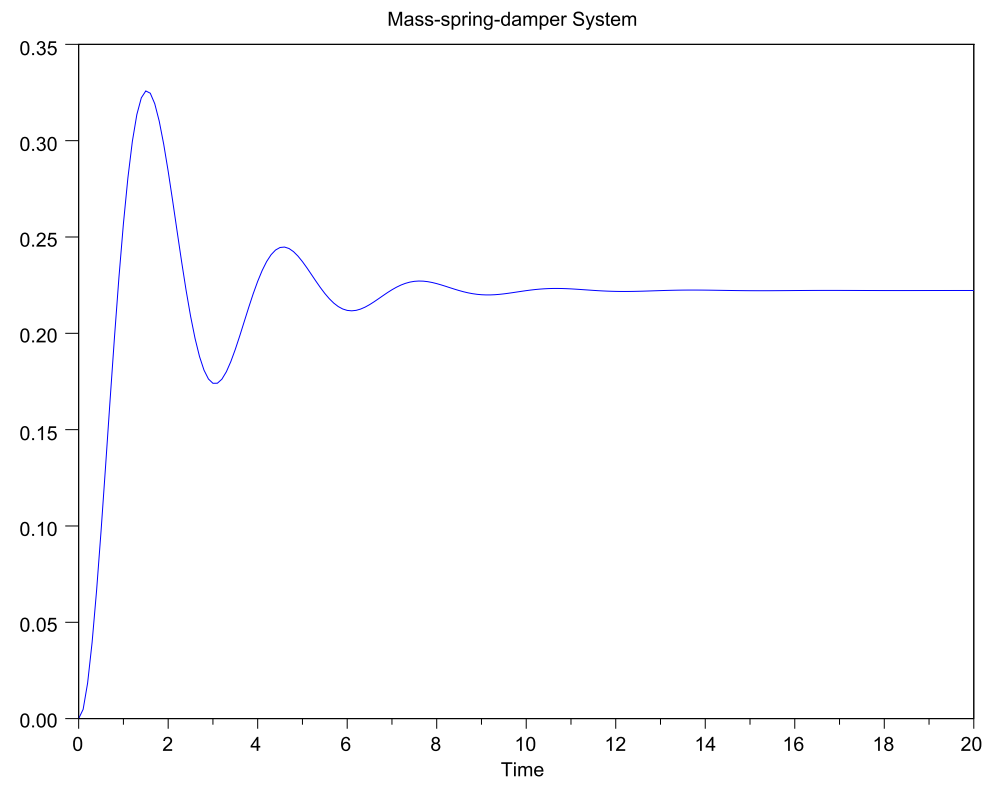

Figure 5: Mass position simulation 
the interval $[-1 ; 1]$ with the following transformation:

$$
\frac{p_{i}-E\left[p_{i}\right]}{\left(b_{i}-a_{i}\right) / 2}, \forall i \in\{1,2,3\}
$$

where $b_{i}$ and $a_{i}$ are, respectively, the maximum and minimum values of the parameter interval and $E\left[p_{i}\right]$ the expectation of $p_{i}$.

2. PC degree

Here, the number of parameters is $n=3$ and the degree used for the polynomials is $d=4$. This degree of expansion was sufficient due to the smoothness of the function, as shown in figure 6 .

\section{PC truncation}

Consequently, the number of terms in the decomposition (the number of coefficients to be calculated) is $M+1=35$, according to (15).

4. PC coefficients

After substituting (45) for $p_{i}$, the multivariate polynomials $\Phi_{j}$ are given by (16):

$$
\Phi_{j}\left(p_{1}, p_{2}, p_{3}\right)=\prod_{k=1}^{3} \phi_{a_{k}^{j}}\left(p_{k}\right)
$$

where $\left|a^{j}\right|=\sum_{k=1}^{3} a_{k}^{j} \leq 4, j=0, \ldots, 34$, and $\phi_{a_{k}^{j}}$ are one-dimensional Legendre polynomials. The 35 coefficients $\alpha_{j}(t)$ are determined with the projection method because the given dynamic system is relatively smooth. Therefore, the coefficients are given by (23). The software Scilab toolbox NISP [35] helps to compute them. The error $y(t)-\sum_{j=0}^{34} \alpha_{j}(t) \Phi_{j}(\mathbf{p})$, represented in figure 6 , is low in the transient and stabilizes in the steady state, showing a good approximation of the output with the PC decomposition. Moreover, figures 7 and 8 show the PDF of the output, at different time instants.

\subsubsection{Sensitivity functions}

The next step is to compute the sensitivity functions for the three parameters according to (33), (34) and (35). Figures 9, 10 and 11 represent, respectively, the first-order, the higher order and the total sensitivity functions.

Several instants of time were chosen to cover the transient and the steady-state of the system: the functions were calculated every 0.2 seconds. For the first seconds of the transient, the most influential parameters on the mass position are obviously the mass and the damper, because they depend on the acceleration 


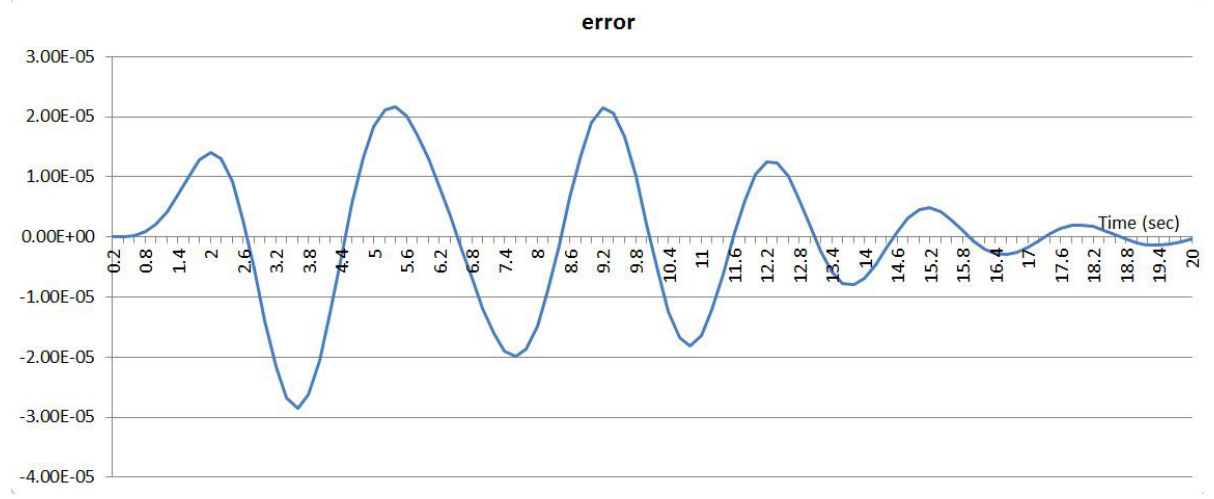

Figure 6: Error between the output and its PC-based approximation

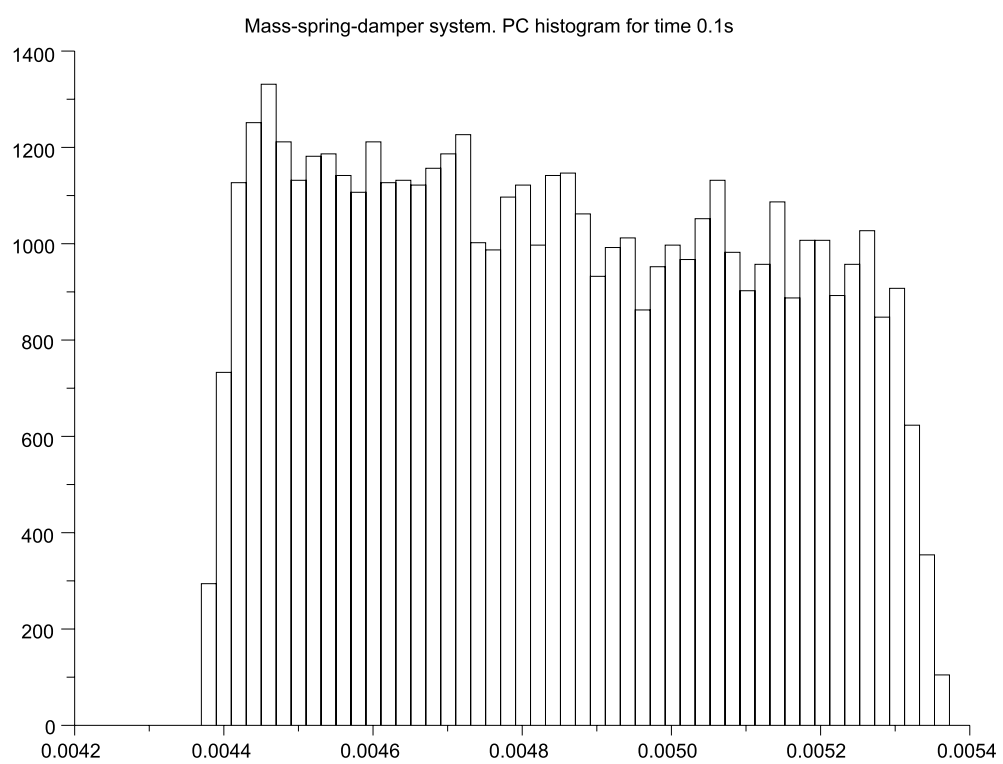

Figure 7: PDF of the output at time $0.1 \mathrm{~s}$ 


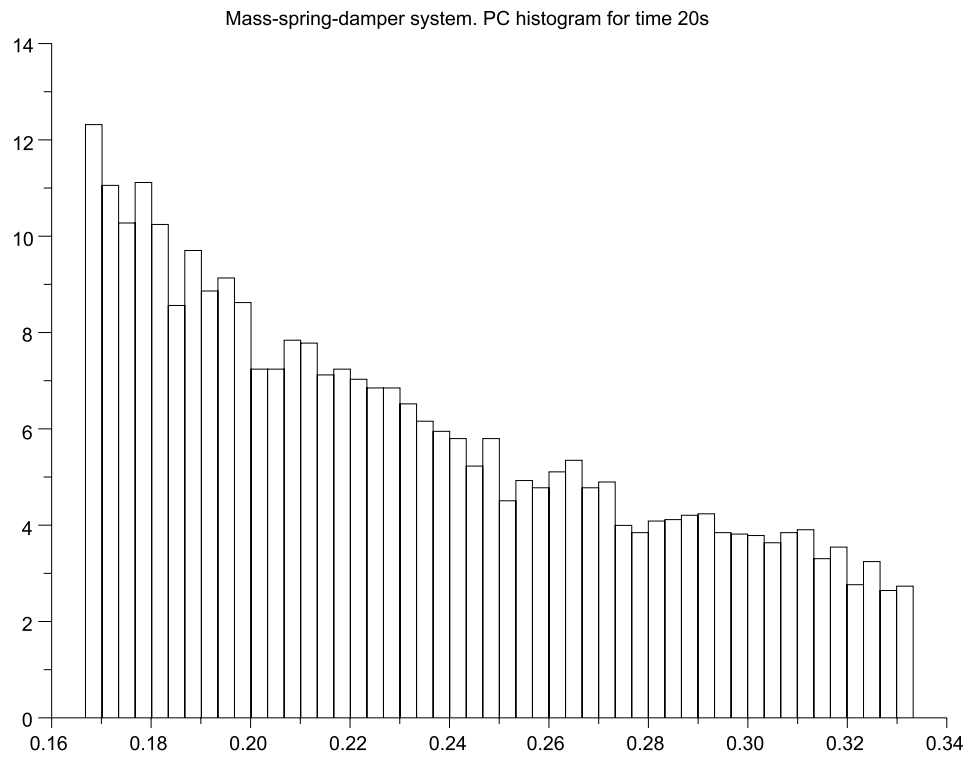

Figure 8: PDF of the output at time 20s

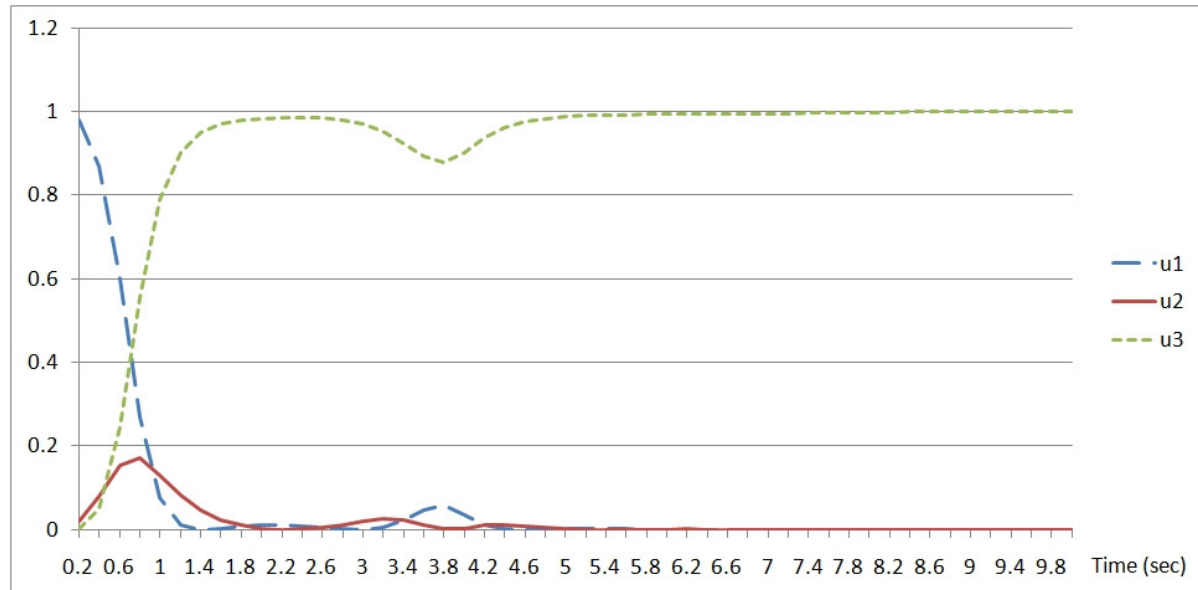

Figure 9: 1st-order sensitivity functions 


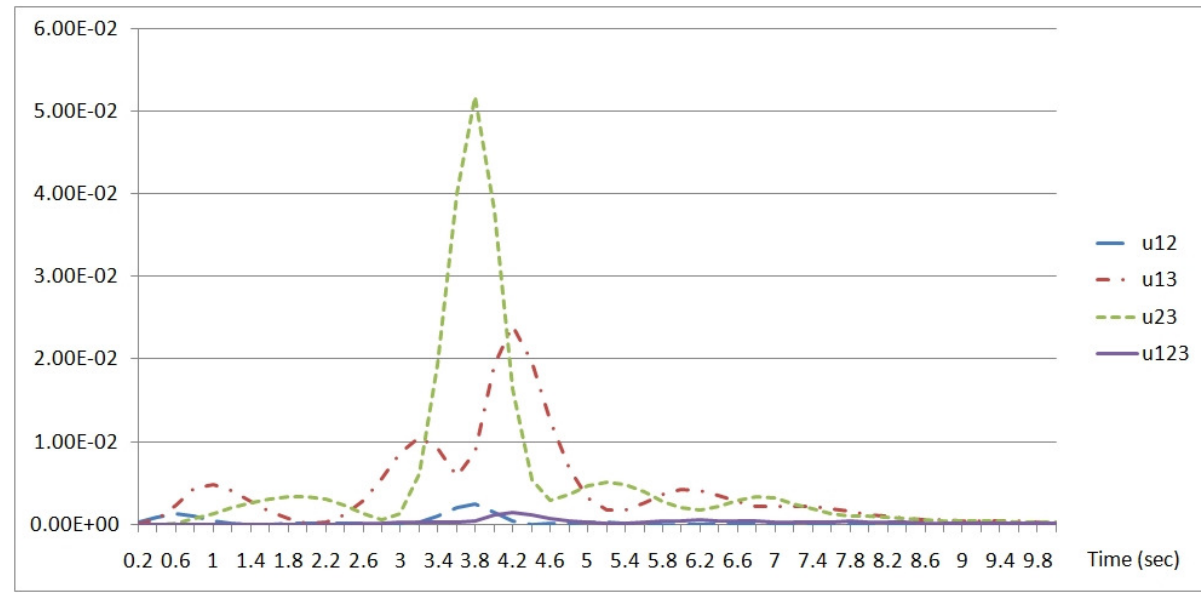

Figure 10: Sensitivity functions of higher order

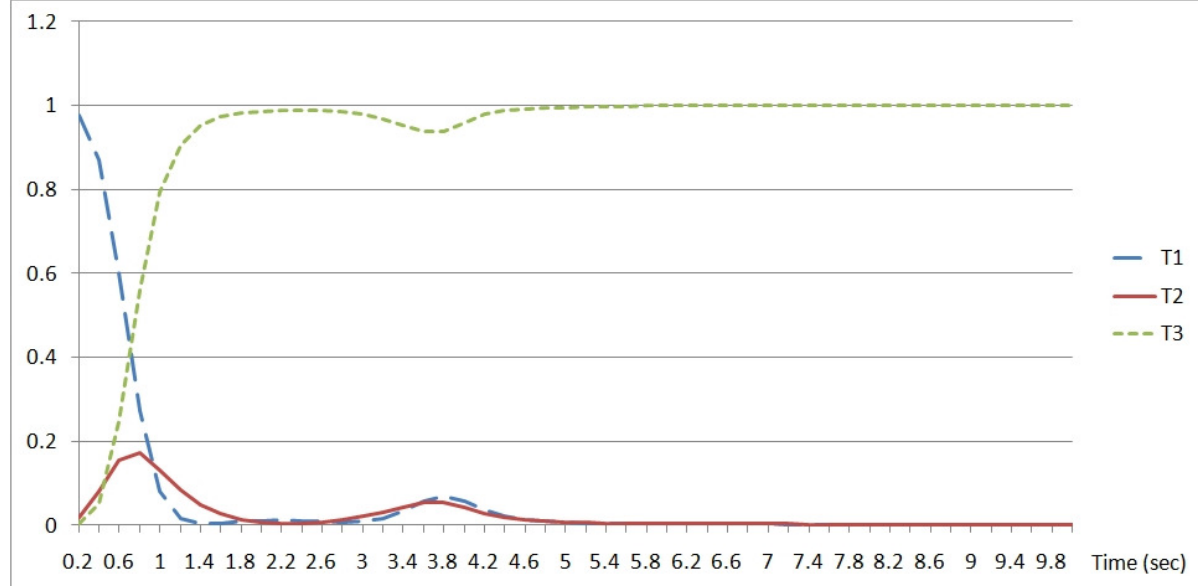

Figure 11: Total sensitivity functions 


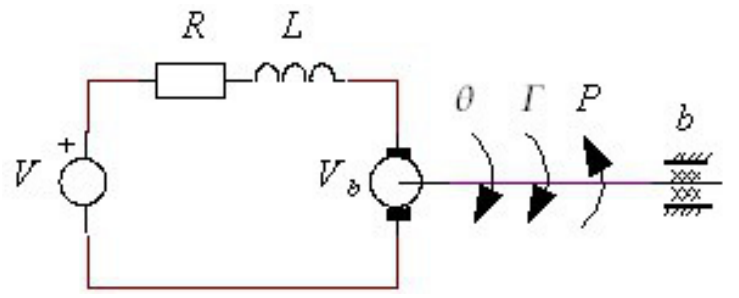

Figure 12: DC motor diagram

and the speed (see figure 9). When the speed reaches its maximum value and the acceleration is zero, approximately at 0.6 seconds, the mass and the spring become equally influent. From second 2 up to the steady state, the spring is clearly the most relevant parameter because the velocity and the acceleration tend to zero. The second order indices $S_{13}$ (mass-spring) and $S_{23}$ (damperspring) appear more clearly in the middle of the simulation (figure 10), when the position is already significant and the velocity and acceleration are still not zero.

\subsection{DC motor system}

Another example of a known dynamic system is the permanent magnet DC motor (figure 12). The parameters of this system are the internal resistance $R$, the inductance $L$, the motor constant $k_{m}$, the counter electromotive constant $k_{a}$, the mechanical inertia $J$ and the mechanical damping $b$. The input is the voltage $V$ applied to the motor and the output is the shaft speed $\omega(t)$.

The torque is given by $\Gamma=k_{m} i(t)$ and the counter electromotive voltage by $V_{b}=k_{a} \omega(t)$. We assume that the motor is unloaded, so the equations of this system are:

$$
\begin{aligned}
V & =R i(t)+L \frac{\mathrm{d} i(t)}{\mathrm{d} t}+k_{a} \omega(t) \\
J \dot{\omega}(t) & =k_{m} i(t)-b \omega(t)
\end{aligned}
$$

Defining the states as $x_{1}(t)=i(t)$ and $x_{2}(t)=\omega(t)$, the state space representation of this system is:

$$
\left[\begin{array}{l}
\dot{x}_{1}(t) \\
\dot{x}_{2}(t)
\end{array}\right]=\left[\begin{array}{cc}
-\frac{R}{L} & -\frac{k_{a}}{L} \\
\frac{k_{m}}{J} & -\frac{b}{J}
\end{array}\right]\left[\begin{array}{l}
x_{1}(t) \\
x_{2}(t)
\end{array}\right]+\left[\begin{array}{c}
\frac{1}{L} \\
0
\end{array}\right] V
$$

Figure 13 represents the evolution of $\omega(t)$, when the step signal $V=9$ is applied for the fixed values $L=0.3, R=15, J=0.2, b=0.2, k_{m}=7.5$ and $k_{a}=3$. 


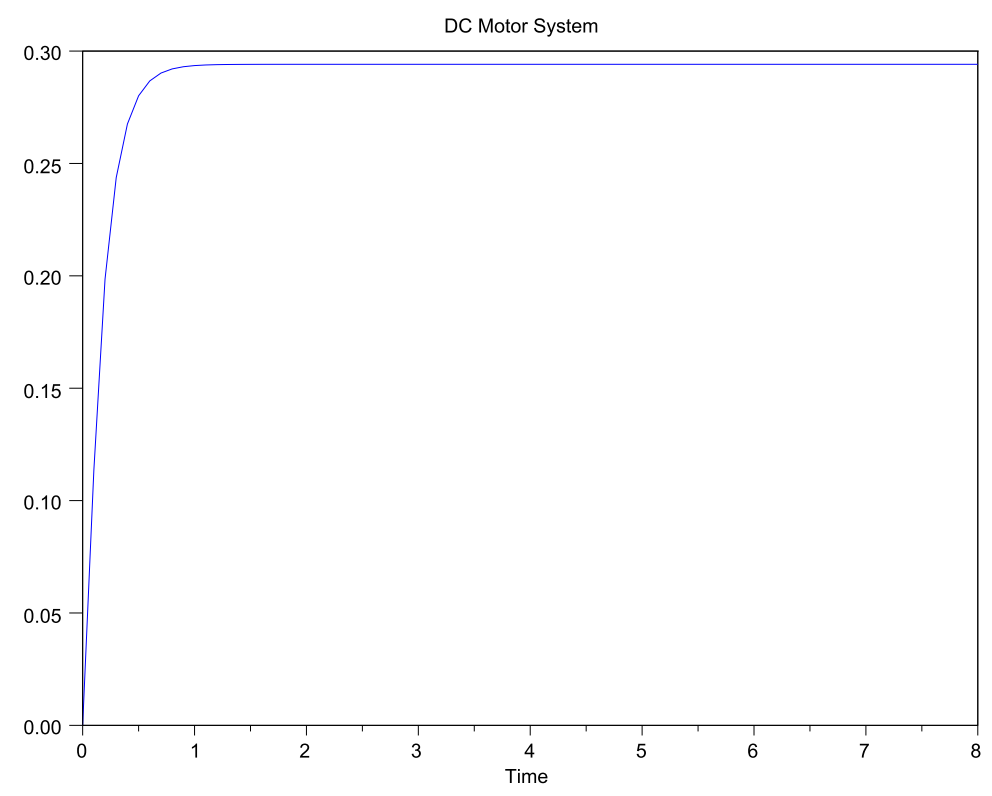

Figure 13: Response of a step signal 


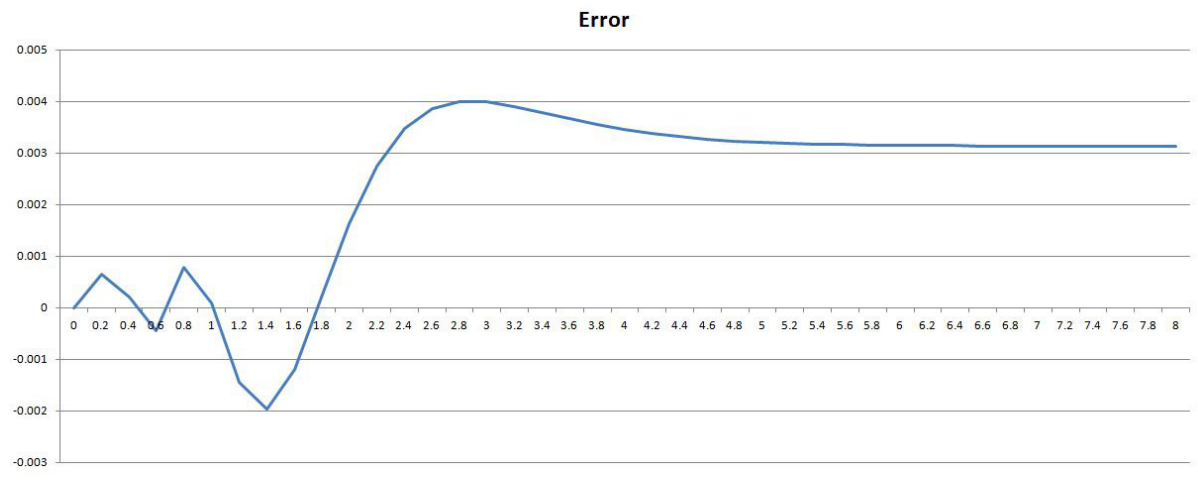

Figure 14: Error between the output and its PC-based approximation

\subsubsection{PC decomposition}

1. Polynomial type

The parameters are considered as independent random variables with a uniform distribution in the intervals $L \in[0.2 ; 0.4], R \in[10 ; 20], J \in$ $[0.2 ; 0.4], b \in[0.1 ; 0.3], k_{m} \in[5 ; 10]$ and $k_{a} \in[1 ; 5]$. Thus, as previously, $\phi_{a_{k}^{j}}$ are Legendre polynomials. Note that the parameters are mapped onto the interval $[-1 ; 1]$, before the PC decomposition.

\section{PC degree}

Here, the number of parameters is $n=6$ and the degree used for the polynomials is $d=5$. This degree is sufficient due to the smoothness of the function, as shown in figure 13. The error between the original system and the PC approximation is obtained to verify the accuracy of the PC expansion, as shown in figure 14 .

\section{PC truncation}

The number of terms in the decomposition is $M+1=462$.

4. PC coefficients

The multivariate polynomials $\Phi_{j}$ are given by (16), with $n=6$ and where $\left|a^{j}\right|=\sum_{k=1}^{6} a_{k}^{j} \leq 5, j=0, \ldots, 461$, and $\phi_{a_{k}^{j}}$ are one-dimensional Legendre polynomials. The error $y(t)-\sum_{j=0}^{461} \alpha_{j}(t) \Phi_{j}(\mathbf{p})$, represented in figure 14 , is less than $0.15 \%$, showing a good approximation of the output with the PC decomposition. 


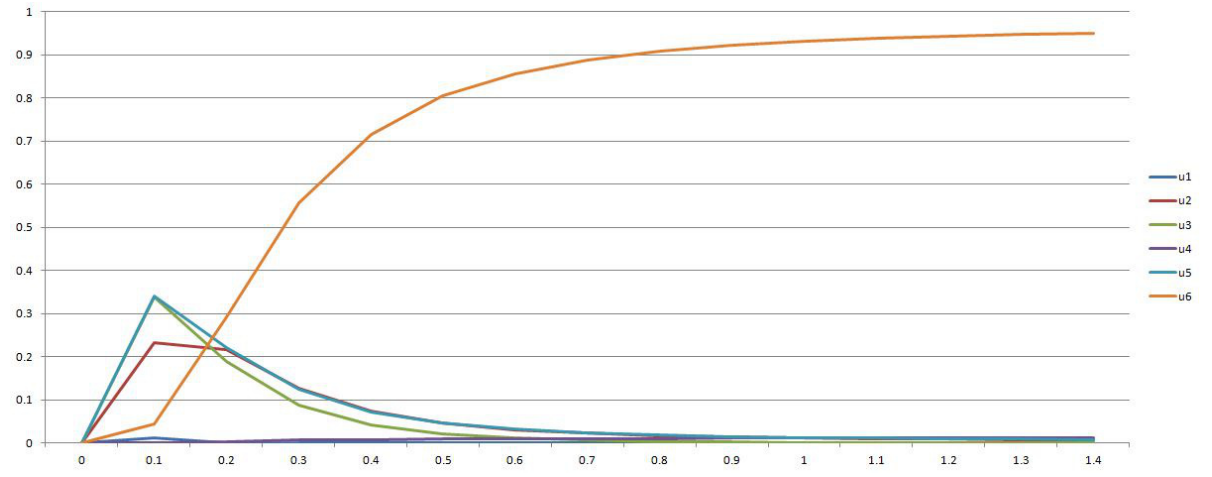

Figure 15: 1st-order sensitivity functions

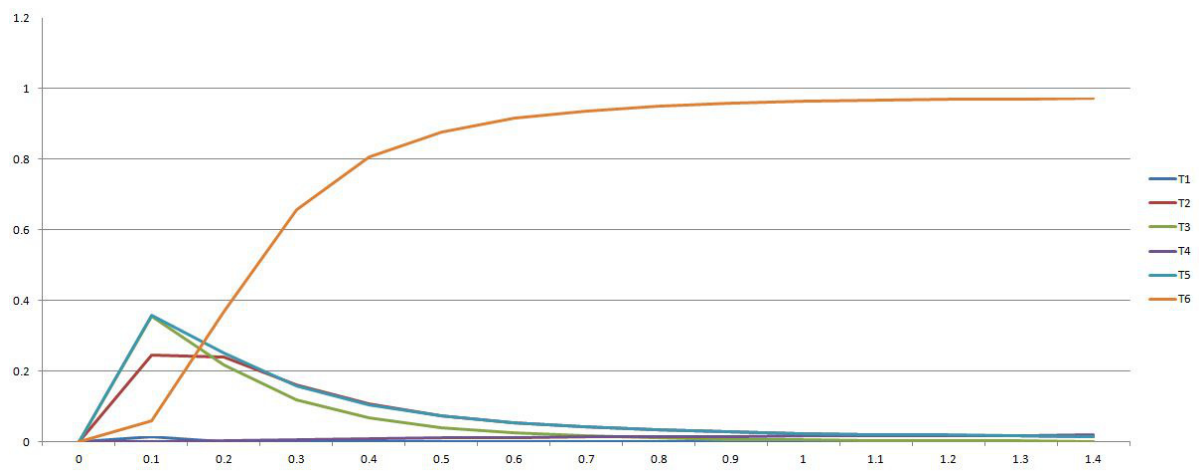

Figure 16: Total sensitivity functions

\subsubsection{Sensitivity functions}

Finally, the sensitivity functions are computed. Figure 15 presents the first order sensitivity functions with $u 1=L, u 2=R, u 3=J, u 4=b, u 5=k_{m}$, $u 6=k_{a}$ and figure 16 presents the total sensitivity functions with $T 1=L$, $T 2=R, T 3=J, T 4=b, T 5=k_{m}, T 6=k_{a}$. The inductance $L$ and the damping $b$ have little importance throughout the simulation. This is because the pole generated by these constants is much faster than the dominant pole. In practice, these parameters can be neglected or settled to their nominal value. The inertia $J$, the resistance $R$ and the motor constant $k_{m}$ are important during the transient. Then, their influence disappears when reaching the steady state. The parameter $k_{a}$ is the most influential parameter in the steady state, since it is a constant which is multiplied by the speed of the shaft.

\section{Conclusion}

This paper has presented the problem of the global sensitivity of dynamic systems which proves important when parametric uncertainty falls within a wide 
range for several parameters and when the sensitivity changes significantly from one operation point to another.

The polynomial chaos expansion is used to calculate the sensitivity indices of the system. The expansion coefficients are calculated either through the technique of regression or projection, which are non-intrusive methods. As the sensitivity of a dynamic system changes over time, the sensitivity indices are calculated at different time instants. In this way, the global sensitivity functions of the dynamic system are obtained.

A major advantage of this approach is that, if the function to be represented with the PC is smooth, the number of coefficients that must be calculated is small. So, computation time is small too, compared with the Monte Carlo simulation.

The approach presents good results for relative simple dynamic models, as presented in this work. Further work will be carried out on more complex models (partial differential equations, for instance) to attest its efficiency and test its accuracy of $\mathrm{PC}$ decomposition in long term.

\section{Acknowledgements}

The authors wish to thank Michael Baudin (Consortium Scilab) for his valuable guidance in the use of software NISP.

\section{References}

[1] G. Blatman, B. Sudret, Sparse polynomial chaos expansion and adaptive stochastic finite element using a regresion approach, Comptes rendus de Mécanique 336 (6) (2008) 518-523.

[2] E. Borgonovo, A new uncertainty importance measure, Reliability Engineering \& System Safety 92 (2007) 771-784.

[3] R. I. Cukier, R. I. Levine, K. E. Shuler, Nonlinear sensitivity analysis of multiparameter model systems, Journal of Computational Physics 26 (1978) 1-42.

[4] H. Frey, S. R. Patil, Identification and review of sensitivity analysis methods, Risk Analysis 22 (3) (2002) 553-578.

[5] A. Saltelli, Sensitivity analysis for importance assessment, Risk Analysis 22 (3) (2002) 579-590.

[6] A. Saltelli, S. Tarantola, F. Campolongo, N. Ratto, Sensitivity Analysis in Practice. A Guide to Assessing Scientific Models, Wiley, 2004.

[7] I. M. Sobol, Sensitivity estimates for nonlinear mathematical models, Mathematical Modelling and Computing Experiments 1 (1993) 407-414. 
[8] T. Turyani, H. Rabitz, Local methods in sensitivity analysis, A. Saltelli, K. Chan, E. M. Scott, John Wiley and Sons, Chichester, 2000.

[9] T. Homma, A. Saltelli, Importance measures in global sensitivity analysis of model output, Reliability Engineering \& System Safety 52 (1) (1996) $1-17$.

[10] A. Saltelli, S. Tarantola, K. Chan, A quantitative model independent method for global sensitivity analysis of model output, Technometrics 41 (1999) 39-56.

[11] E. Borgonovo, W. Castaings, S. Tarantola, Model emulation and moment-independent sensitivity analysis: An application to environmental modelling, Environmental Modelling \& Software (0) (2011) -. doi:10.1016/j.envsoft.2011.06.006.

[12] M. Ratto, A. Pagano, Using recursive algorithms for the efficient identification of smoothing spline anova models, AStA Advances in Statistical Analysis 94 (4) (2010) 367-388.

[13] J. Jacques, C. Lavergne, N. Devictor, Sensitivity analysis in presence of model uncertainty and correlated inputs, Reliability Engineering \& System Safety 91 (2006) 1126-1134.

[14] T. A. Mara, S. Tarantola, Application of global sensitivity analysis of model output to building thermal simulations, Journal of Building Simulation 1 (2008) 290-302.

[15] M. D. McKay, J. D. Morrison, S. C. Upton, Evaluating prediction uncertainty in simulation models, Computer Physics Communications 117 (1-2) (1999) 44-51.

[16] N. Wiener, The homogeneous chaos, American Journal of Mathematics 60 (4) (1938) 897-936.

[17] R. Ghanem, J. Red-Horse, Propagation of probabilistic uncertainty in complex physical systems using a stochastic finite element approach, Physica D 133 (1999) 137-144.

[18] R. Ghanem, P. Spanos, Stochastic finite elements - A spectral approach, Springer Verlag, 1991.

[19] T. Crestaux, O. L. Maitre, J. Martinez, Polynomial chaos expansion for sensitivity analysis, Reliability Engineering \& System Safety 94 (2009) 11611172 .

[20] B. Sudret, Global sensitivity analysis using polynomial chaos expansion, Reliability Engineering \& System Safety 93 (2008) 964-979. 
[21] G. Blatman, B. Sudret, Efficient computation of global sensitivity indices using sparse polynomial chaos expansions, Reliability Engineering \& System Safety 95 (11) (2010) 1216 - 1229.

[22] G. T. Buzzard, Global sensitivity analysis using sparse grid interpolation and polynomial chaos, Reliability Engineering \& System Safety (0) (2011) -. doi:10.1016/j.ress.2011.07.011.

[23] S. Rahman, Global sensitivity analysis by polynomial dimensional decomposition, Reliability Engineering \& System Safety 96 (7) (2011) 825 - 837.

[24] D. J. W. D. Pauw, P. A. Vanrolleghem, Practical aspects of sensitivity function approximation for dynamic models, Mathematical and Computer Modelling of Dynamical Systems 12 (2006) 395-414.

[25] M. Perry, H. P. Wynn, R. A. Bates, Principal components analysis in sensitivity studies of dynamic systems, Probabilistic engineering mechanics 21 (4) (2006) 454-460.

[26] J. Witteveen, H. Bijl, Modeling arbitrary uncertainties using GramSchmidt polynomial chaos, in: 44th AIAA Aerospace Sciences Meeting and Exhibit, 2006.

[27] D. Xiu, G. Karniadakis, The Wiener-Askey polynomials chaos for stochastic differential equations, Journal of Scientific Computing 26.

[28] D. Xiu, G. Karniadakis, Modeling uncertainty in flow simulations via generalized polynomial chaos, Journal of Computational Physics 187 (1) (2002) 137-167.

[29] R. Field, Numerical methods to estimate the coefficients of the polynomial chaos expansion, in: 15th ASCE Engineering Mechanics Conference, 2002.

[30] M. Berveiller, B. Sudret, M. Lemaire, Stochastic finite element: a non intrusive approach by regression, European Journal of Computational Mechanics 15 (2006) 81-92.

[31] B. Sudret, M. Berveiller, M. Lemaire, Application of a stochastic finite element procedure to reliability analysis, European Journal of Computational Mechanics 15 (7-8) (2006) 825-866.

[32] M. Eldred, Recent advances in non-intrusive polynomial chaos and stochastic collocation methods for uncertainty analysis and design, Tech. rep., 50th AIAA/ASME/ASCE/AHS/ASC Structures, Structural Dynamics, and Materials Conference (2009).

[33] G. Agusti, G. I. Schueller, M. Ciampoli (Eds.), Non linear non intrusive stochastic finite element method - application to a fracture mechanics problem, 9th International Conference on Structure, Safety and Reliability, Millpress, Roma, Italy, 2005. 
[34] B. Sudret, Uncertainty propagation and sensitivity analysis in mechanical models - Contributions to structural reliability and stochastic spectral methods, habilitation à diriger des recherches, Université Blaise Pascal, Clermont-Ferrand, France (2007).

[35] M. Baudin, J. Martinez, Polynômes de chaos sous Scilab via librarie NISP, in: 42èmes Journées de Statistique, 2010. 